\title{
Research and Analysis on the Supporting Structure of the Second Lining of the Tunnel in the Phyllite Stratum
}

\author{
WeiZhiquan $^{1}$, HuangBaisheng ${ }^{*}$, YangLu ${ }^{1}$, WeiYonghao ${ }^{1}$, QiuJianqiao ${ }^{1}$ \\ ${ }^{1}$ Jiangxi Provincial Expressway Investment Group Co., Ltd., Nanchang 330200
}

\begin{abstract}
The reasonable construction of the secondary lining structure of the tunnel is an important link to ensure the stability of the surrounding rock of the tunnel. Taking a phyllite stratum tunnel project in Jiangxi as the background, the Flac3D finite difference software was used to numerically simulate and analyze the supporting structure of the secondary lining. The impact of support timing on surrounding rock stress. The calculation results show that with the progress of the construction step, the main period of the displacement of the vault is after the excavation of the upper section of the tunnel before the upper section support; The plastic zone of the surrounding rock changes obviously, especially above the tunnel, and finally the plastic zone develops to the ground; the stress of the surrounding rock shows a decreasing trend with the increase of the time of the secondary lining. The research results can provide certain guiding significance for the construction of the second lining of the tunnel under similar geological conditions.
\end{abstract}

\section{Introduction}

In tunnel engineering, the basic concept of the New Austrian Method is to consider the surrounding rock as part of the tunnel supporting system and give full play to the supporting capacity of the surrounding rock itself. Therefore, it is necessary to carry out research on the interaction relationship between "supporting and surrounding rock", and then select a reasonable time of support to ensure the smooth implementation of the new Austrian method of construction. Sung et al ${ }^{[1]}$. studied the optimal support design for tunnel excavation in weak rock masses based on the principles of the New Austrian Method. L.R.Alejano et al ${ }^{[2]}$, based on the strain softening model of the surrounding rock, and calculated the support timing of the secondary lining based on this. Liu Beishi et $\mathrm{al}^{[3]}$.deduced that different surrounding rock levels correspond to different tunnel support ranges based on the elastoplastic theory. Xie Feng et $\mathrm{a}^{[4]}$, analyzed the supporting timing of the secondary lining based on the rheological characteristics of the surrounding rock.

Based on a phyllite tunnel project in Jiangxi, this article analyzes the influence of each construction step of tunnel excavation on the deformation of surrounding rock, and analyzes the changes in the axial force of the secondary lining under two working conditions, and discusses how to grasp the support of the secondary lining The opportunity to save the support costs as much as possible while ensuring the safety of the project. The research results can provide a useful reference for the design and construction of similar projects.

\section{Project Overview}

The tunnel is located in a low-mountainous landform of structural denudation. The mountain is generally in the north-south direction, with branch veins and branches, and the terrain is undulating. The surface of the tunnel is the Quaternary Holocene residual slope $Q_{4}^{e l+d l}$ silty clay, and the underlying bedrock is Yanshanian $\left(\mathrm{r}_{4}\right)$ granite. The tunnel entrance is well-developed with joints and fissures. The rock mass is relatively broken and has a massive structure. The stability of the rock mass is poor. The tunnel body is mainly located in Yanshanian $\left(r_{4}\right)$ granite. The joints are generally developed and the rock mass is relatively complete. The fissures, which intersect with the tunnel body at a large angle, have a certain influence on the stability of the tunnel surrounding rock structure.

\section{Numerical Simulation}

\subsection{Calculation model}

According to the actual working conditions of the tunnel, the $3 \mathrm{D}$ tunnel excavation problem is simplified into a $2 \mathrm{D}$ plane model, and the right section of the tunnel is selected for the analysis section. The specific pile number is YK7+709.00. The calculation model and numerical grid analysis model are shown in Figure 1 and Figure 2. According to the survey and design data, the physical and mechanical parameters of each surrounding rock formation are shown in Table 1. Because the stress

* Corresponding author:962840920@qq.com 
distribution is only affected in a certain range during tunnel construction, it is generally 3 to 5 times the diameter of the tunnel ${ }^{[5-6]}$. This model takes the upper boundary to the ground surface, the left and right boundaries are 5 times the span of the tunnel, and the left and right boundaries are each $60 \mathrm{~m}$. The lower boundary is 5 times the height of the tunnel, and the lower boundary is $54 \mathrm{~m}$. The model size is $60 \mathrm{~m} \times 80 \mathrm{~m}$ (length $\times$ height).

Table 1 Calculation parameters of elastoplastic mode

\begin{tabular}{cccccc}
\hline Surrounding rock & $E / \mathrm{GPa}$ & $\mu$ & $C / \mathrm{kPa}$ & $\varphi /{ }^{\circ}$ & $\gamma / \mathrm{kN} \cdot \mathrm{m}^{3}$ \\
\hline$\delta_{-3-1}$ & 0.02 & 0.41 & 18.9 & 20.9 & 19.3 \\
$\delta_{-1-1}$ & 1.4 & 0.32 & 220 & 28.0 & 21.8 \\
$\delta_{-1-2}$ & 3.2 & 0.31 & 460 & 30.0 & 24.3 \\
$\delta_{-1-3}$ & 5.1 & 0.33 & 790 & 35.0 & 26.2 \\
\hline
\end{tabular}

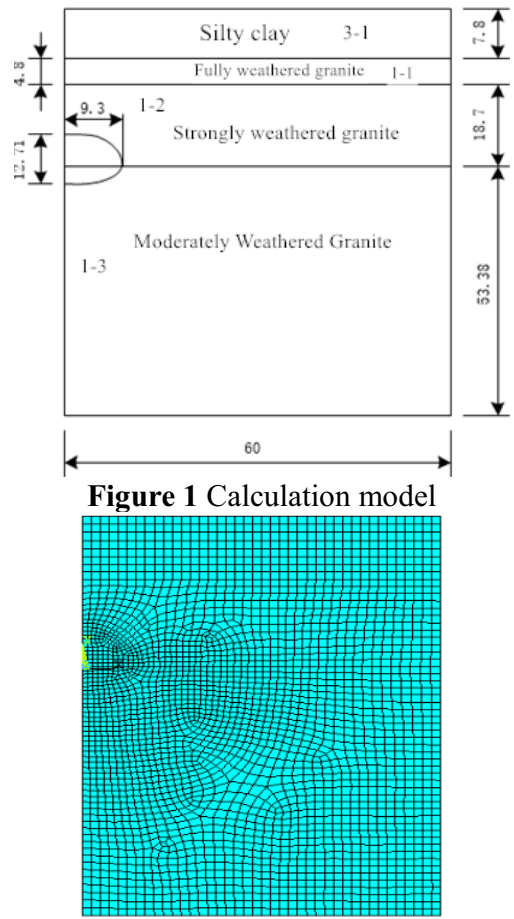

Figure 2 Numerical grid analysis model

\subsection{Calculation conditions}

According to the design data, the tunnel adopts composite primary support anchor shotcrete + section steel and grid arch bracket. The initial support parameters are as follows: The full section is equipped with double-layer composite spray net, the first layer of concrete thickness is $26 \mathrm{~cm}+$ double-layer $\Phi 6.5 \mathrm{~mm}$ steel mesh, grid spacing is $20 \mathrm{~cm} \times 20 \mathrm{~cm}, \mathrm{I} 20 \mathrm{~b}$ steel arch frame, spacing $0.75 \mathrm{~m}$; The initial support of the second layer is a $20 \mathrm{~cm}$ thick composite shotcrete and grid arch frame with a spacing of $0.75 \mathrm{~m}$; the arch and wall are equipped with hollow grouting anchor rods, the anchor rod length is $4.0 \mathrm{~m}$, and the spacing is $0.75 \mathrm{~m} \times 1.0 \mathrm{~m}$; the reserved deformation is $17 \mathrm{~cm}$. The secondary lining is $\mathrm{C} 30$ cast-in-place reinforced concrete with a thickness of $45 \mathrm{~cm}$.

Divide the entire tunnel excavation into 6 calculation steps, without considering the "spatial effect" of the long bench construction method, the specific calculation steps are as follows: Step 1, excavation of the right wall; Step 2, support of the right wall; third Step, the excavation of the upper step; Step 4, the support of the upper section; Step 5, the excavation of the lower section; Step 6, the full-section two lining construction.

\section{Analysis of calculation results}

According to the changes in the stress field of surrounding rock in Figure 3 and Figure 4, it can be seen that after the side wall is excavated, the stress at the end of the side wall increases from the original $0.74 \mathrm{MPa}$ to $1.05 \mathrm{MPa}$. After the initial support in the second step, the left end of the rock formation has reached 1.52MPa due to continuous stress growth. After the excavation of the upper section in the third step, the stress of the surrounding rock of the vault changed sharply, with a minimum value of $0.21 \mathrm{MPa}$. After the upper section support in the fourth step, the surrounding rock stress changes relatively slowly. After the excavation of the lower end face, the stress continued to increase only near the right arch foot due to the stress concentration effect. After the last two linings are supported, the surrounding rock stress is basically in a stable state, but continues to increase with the influence range.

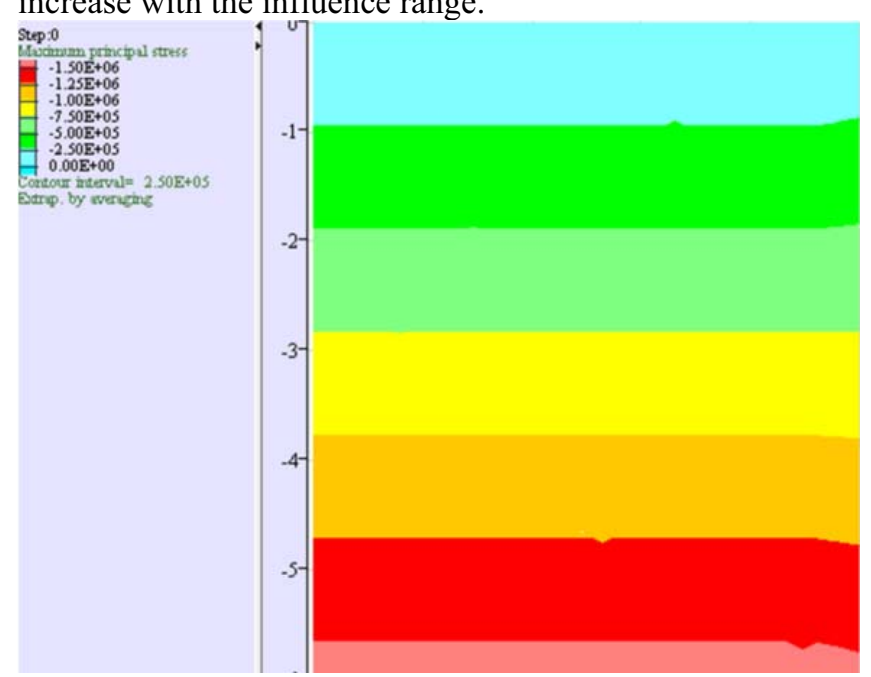

Figure 3 Initial stress distribution diagram of surrounding rock 


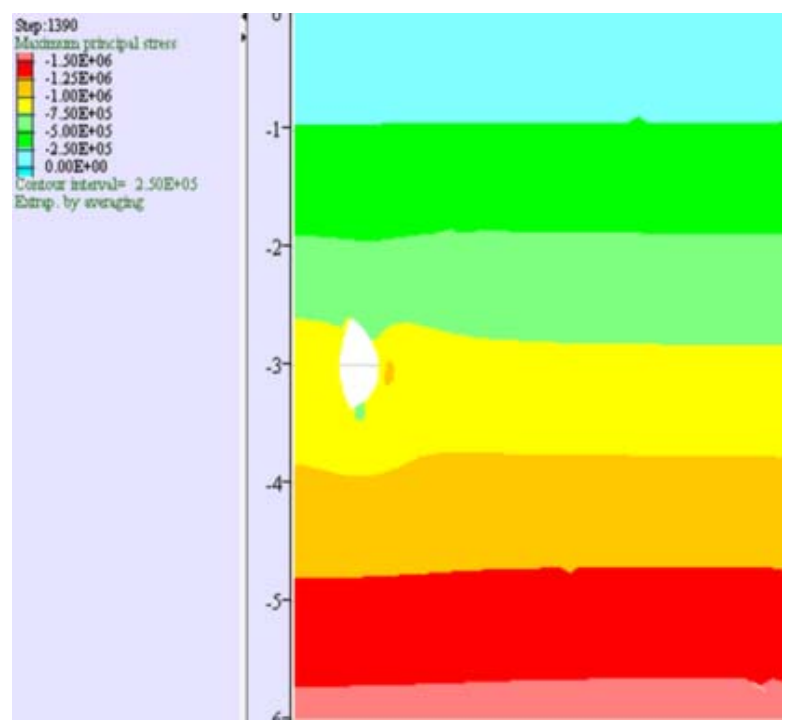

(a) After excavation of the right wall
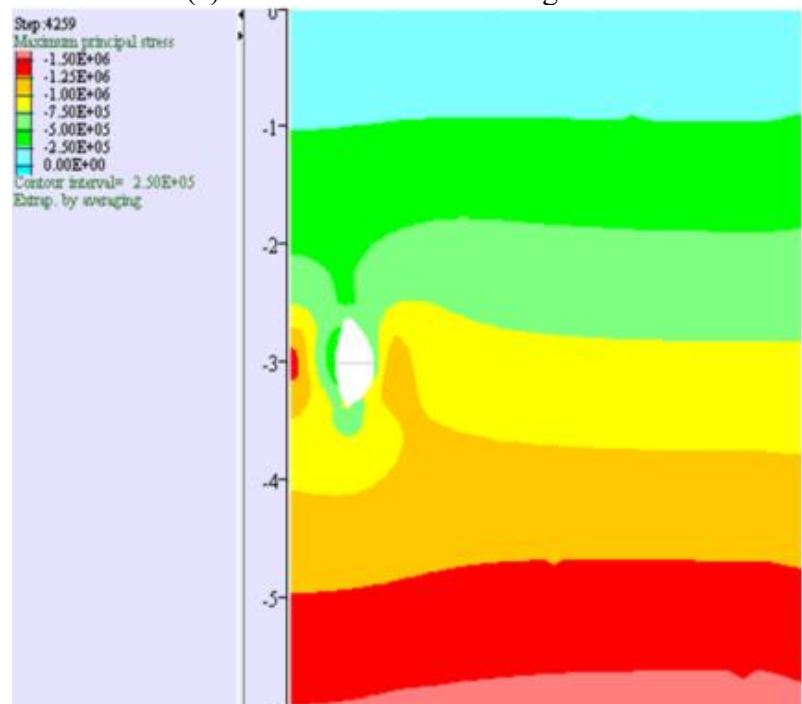

(b) After the right wall is supported

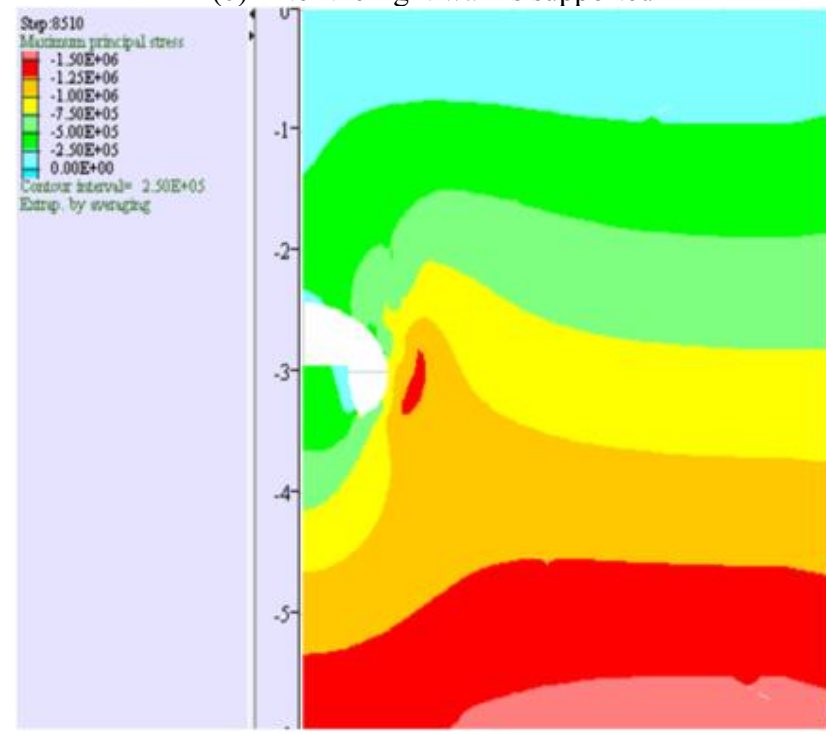

(c) After excavation of the upper section
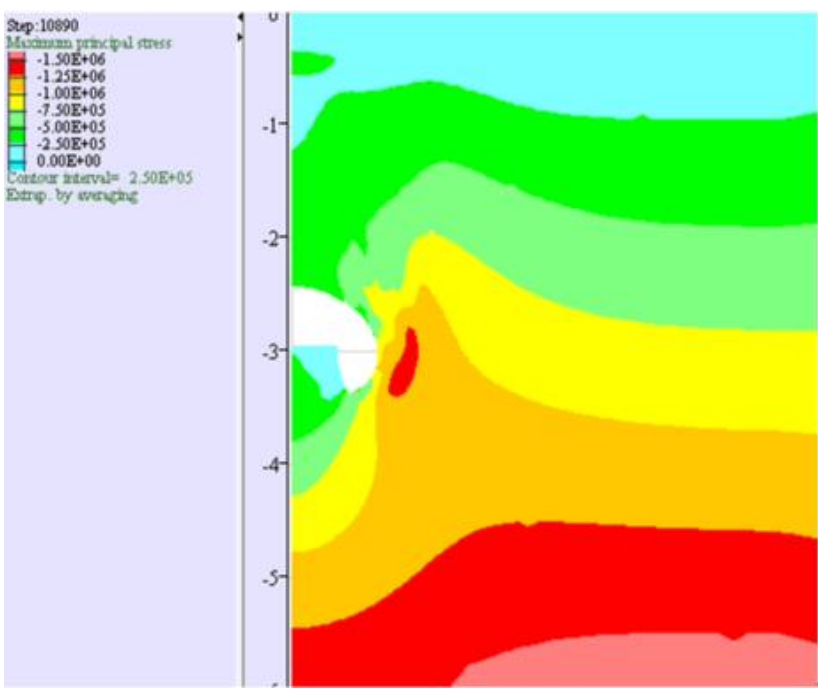

(d) After the upper section support

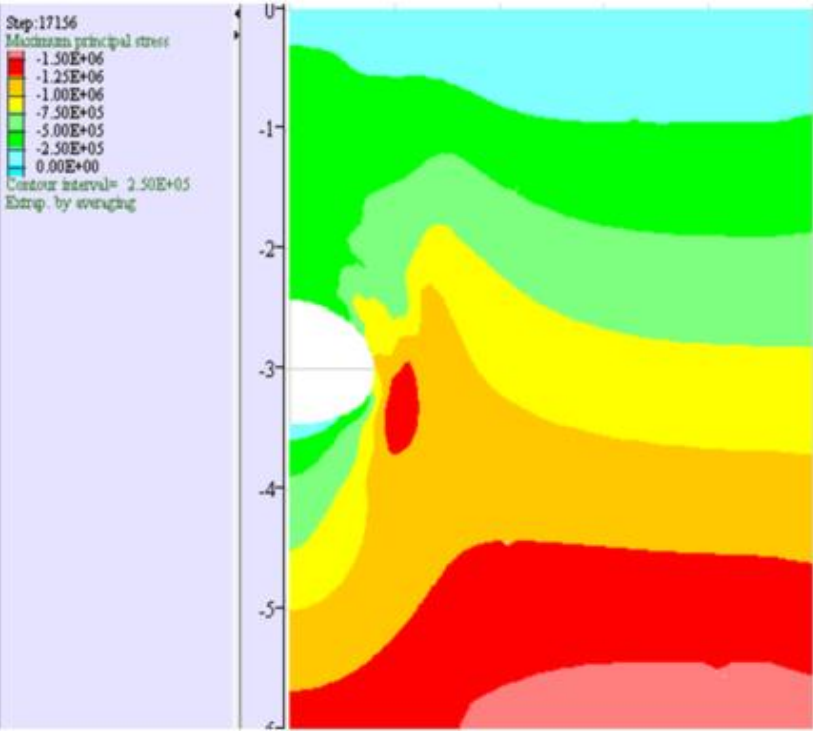

(e) Excavation of the lower section

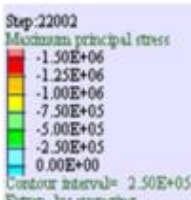

(atres

to

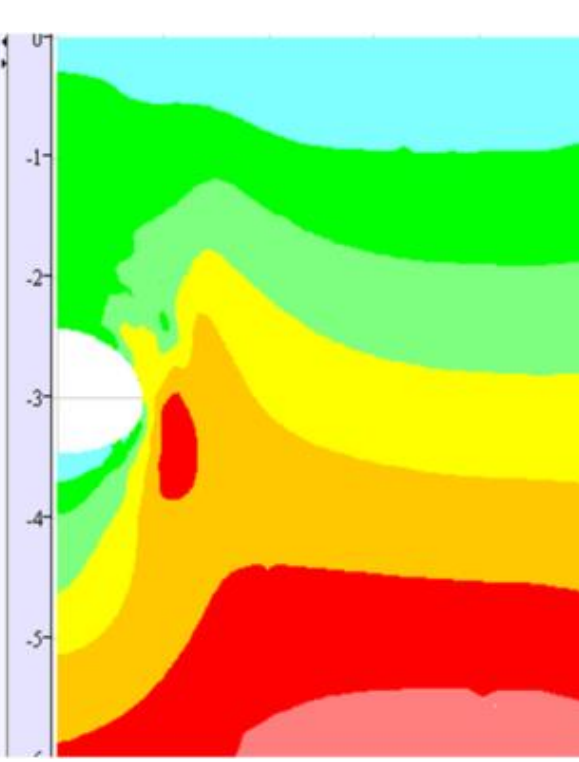

(f) The secondary lining support is closed

Figure 4 Stress distribution diagram of surrounding rock

$$
\text { of tunnel }
$$

In order to analyze the influence of the supporting 
timing of the second lining on the stress of the surrounding rock, the second lining was constructed at 1D and 2D from the tunnel face, and the stress of the second lining was calculated numerically. Considering that this model is a two-dimensional model, in the numerical simulation, the space conditions of these two working conditions are converted into time conditions, that is, assuming that the tunnel construction footage is $2 \mathrm{~m} / \mathrm{d}$, it is converted into 6 days of excavation (1D), after 18 days (2D), the secondary lining support is carried out, and then the axial forces of the secondary lining surrounding rock after 10 days, 20 days, and 30 days under these four conditions are analyzed.

By comparing the above two working conditions, it can be seen that: The shaft force of the second lining is the maximum in the three time periods when the working condition 1 is located, and with the increase of the time when the second lining is applied, the axial force of the second lining shows a decreasing trend. When the maximum time is 30 days, they are $2.43 \mathrm{MPa}$ and 1.46 $\mathrm{MPa}$ respectively; it shows that after the support time is pushed, the surrounding rock release stress increases, and the stress imposed on the secondary lining structure by the surrounding rock decreases after the support. The structural safety is favorable;

\section{Conclusion}

(1) With the progress of the construction step, after the excavation of the upper section of the tunnel to before the upper section support is the main period of the vault displacement, which accounts for about $80 \%$ of the total displacement of the vault; the plastic zone of the surrounding rock changes significantly, especially Above the tunnel, the plastic zone eventually develops to the ground, while the invert and side walls take the rock mass plastic zone to develop within a small area. To ensure that the anchor rod plays a supporting role, it needs to pass through the plastic zone, and it is recommended that the anchor rod at the vault should be appropriate Lengthen to $5 \mathrm{~m}$.

(2) The second lining should be selected at an appropriate time to make full use of the role of the primary support and the second lining to support the surrounding rock.

\section{Acknowledgment}

This research was financially supported by the Science and technology project of Jiangxi Provincial Transportation Department (2015C0067)

\section{References}

1. Choi S O, Shin H S . stability analysis of a tunnel excavated in a weak rock mass and the optimal supporting system design[J]. International Journal of Rock Mechanics and Mining Sciences, 2004, 41(3):537-0.

2. Alejano L R, Alfonso Rodríguez-Dono, María Veiga. Plastic radii and longitudinal deformation profiles of tunnels excavated in strain-softening rock masses[J]. Tunnelling and Underground Space Technology incorporating Trenchless Technology Research, 2012, 30(none):169-182.

3. Liu Pishi, Fang Jianqin. Application of elastoplastic analytical method to determine the reasonable time of tunnel support[J]. Highway, 2009(12):186-188.

4. Xie Feng, Jiang Shuping, Li Jianjun. Study on the supporting time of secondary lining of creeping surrounding rock tunnel[J]. Chinese Journal of Underground Space and Engineering, 2006, 2(5): 805-808.

5. Galli G, Grimaldi A, Leonardi A. Three-dimensional modelling of tunnel excavation and lining $[\mathrm{J}]$. Computers and Geotechnics 2004,31:171-183

6. Pan YW, Dong JJ. TIME-DEPENDENT TUNNEL CONVERGENCE .1. FORMULATION OF THE MODEL[J]. International Journal of Rock Mechanics and Mining Sciences \& Geomechanics Abstracts 1991,28:469-475. 